\title{
Malignant Renal Pelvis Neoplasm
}

National Cancer Institute

\section{Source}

National Cancer Institute. Malignant Renal Pelvis Neoplasm. NCI Thesaurus. Code C7525.

A primary or metastatic malignant neoplasm that affects the renal pelvis. 